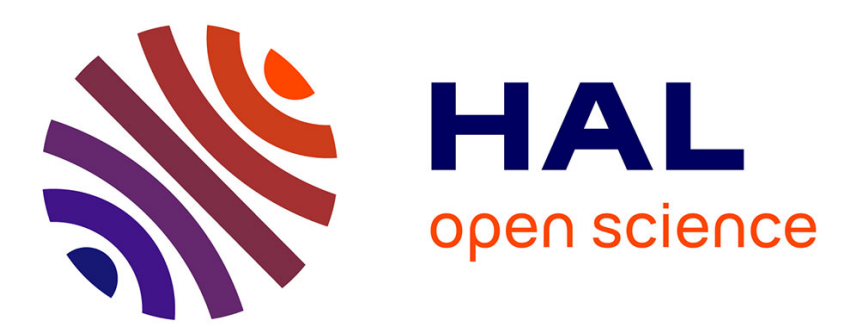

\title{
Etude des résonances de l'hélium par recul de l'atome excité par impact électronique dans un faisceau supersonique
}

\author{
A. Defrance, M. Hagene, D. Pasquerault
}

\section{- To cite this version:}

A. Defrance, M. Hagene, D. Pasquerault. Etude des résonances de l'hélium par recul de l'atome excité par impact électronique dans un faisceau supersonique. Journal de Physique Lettres, 1984, 45 (9), pp.427-432. 10.1051/jphyslet:01984004509042700 . jpa-00232365

\section{HAL Id: jpa-00232365 https://hal.science/jpa-00232365}

Submitted on 1 Jan 1984

HAL is a multi-disciplinary open access archive for the deposit and dissemination of scientific research documents, whether they are published or not. The documents may come from teaching and research institutions in France or abroad, or from public or private research centers.
L'archive ouverte pluridisciplinaire HAL, est destinée au dépôt et à la diffusion de documents scientifiques de niveau recherche, publiés ou non, émanant des établissements d'enseignement et de recherche français ou étrangers, des laboratoires publics ou privés. 
Classification

Physics Abstracts

$34.80 \mathrm{D}-47.40 \mathrm{~K}$

\title{
Etude des résonances de l'hélium par recul de l'atome excité par impact électronique dans un faisceau supersonique
}

\author{
A. Defrance, M. Hagene et D. Pasquerault \\ Département de Physique Atomique et Moléculaire, Université de Rennes I, \\ 35042 Rennes Cedex, France
}

(Reçu le 27 janvier 1984, accepté le 6 mars 1984)

\begin{abstract}
Résumé. - Nous présentons une méthode de mesure indirecte de la diffusion inélastique d'électrons sous grands angles. Cette méthode est basée sur la mesure du recul d'atomes d'un faisceau supersonique excités par choc électronique et détectés dans des états métastables. Nous appliquons cette méthode à l'étude des résonances de l'hélium. Nous montrons que l'état résonnant $\mathrm{He}^{-* * *}\left(2 \mathrm{~s}^{2} 2 \mathrm{p}\right)^{2} \mathrm{P}$ se manifeste par une augmentation de la section efficace de rétrodiffusion inélastique des électrons.

Abstract. - A method giving an indirect measurement of the inelastic scattering of electrons at large angles is described. This method is based on the recoil measurement of atoms in a supersonic beam excited by electron impact and detected in metastable states. This method has been applied to the study of helium resonances. We have found that the resonant state $\mathrm{He}^{-* * * *}\left(2 \mathrm{~s}^{2} 2 \mathrm{p}\right)^{2} \mathrm{P}$ manifests itself as an increase in cross section for the inelastic backscattering of electrons.
\end{abstract}

\section{Introduction.}

Dans les expériences d'excitation des atomes par collision électronique, l'impulsion transférée à l'atome par le choc, accompagné de la diffusion (ou de l'éjection) d'un électron, n'est pas négligeable. Le vecteur vitesse initiale, d'origine thermique, de l'atome subit une modification en direction et en module. Cette propriété a déjà été utilisée sur le potassium [1].

Le recul de l'atome permet, dans certaines conditions, de mesurer de manière indirecte la diffusion des électrons, en particulier, dans des directions difficilement accessibles à la mesure directe : à $0^{\circ}$ (aveuglement du détecteur par le flux direct) et surtout vers $180^{\circ}$ (encombrement), ou plus généralement, lorsque l'électron diffusé est perturbé par un phénomène quelconque (champ électrique ou magnétique par exemple).

Les inconvénients majeurs sont dus :

1) à l'introduction d'une fonction d'appareil importante due en particulier à la dispersion initiale des vitesses lors de l'utilisation d'un jet de four.

2) aux difficultés de détection.

L'utilisation d'un faisceau supersonique présentant une faible dispersion des vitesses en direction et en module, permet de réduire la fonction d'appareil. L'étude d'atomes excités dans un état métastable ou aboutissant à un état métastable facilite leur détection après excitation et déviation. 
Nous utilisons cette méthode pour étudier les deux résonances bien connues de l'hélium, $\mathrm{He}^{-* * * 2} \mathrm{P}$ à $57,2 \mathrm{eV}$ et $\mathrm{He}^{-* * * 2} \mathrm{D}$ à $58,3 \mathrm{eV}$. Les mesures antérieures effectuées de différentes manières par détection des raies de l'hélium [2], des électrons diffusés [3], ou des métastables [4], ont montré le couplage prépondérant de ces états discrets avec le groupe de continuums [hélium simplement excité + électron diffusé] (groupe ${ }^{*}$ )

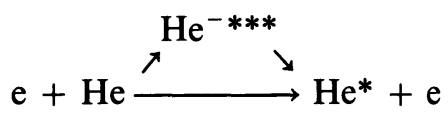

Le comportement angulaire est remarquable, les mesures de la polarisation linéaire des raies de l'hélium faites au laboratoire [5] ont permis de préciser que l'interaction dominante était pour $L>0$ :

$$
\mathrm{He}^{-* * *} / \mathrm{He}^{*}(L)+\mathrm{e}\left(l_{\min }\right)
$$

$l_{\min }$ signifie un changement angulaire minimum des électrons diffusés par rapport à l'axe de quantification (axe des $z$ ). En effet, les niveaux $\mathrm{He}^{-* * *}$ sont préparés dans des états $M_{L}=0$ :

$$
\mathrm{e}\left(l_{z}=0\right)+\operatorname{He}^{1}{ }^{1} \mathrm{~S} \rightarrow \mathrm{He}^{-* * * 2} \mathrm{~L}\left(M_{L}=0\right) .
$$

Ces états agissent fortement sur les continuums mettant en jeu une variation minimum du moment angulaire. On note comme interactions importantes :

$$
\mathrm{He}^{-* * * 2} \mathrm{P}\left(M_{L}=0\right) / \mathrm{He}^{*} 3{ }^{3} \mathrm{P}\left(M_{L}=0\right)+\mathrm{e}(l=0)
$$

et surtout

$$
\mathrm{He}^{-* * * 2} \mathrm{D}\left(M_{L}=0\right) / \mathrm{He}^{*} 3{ }^{3} \mathrm{D}\left(M_{L}=0\right)+\mathrm{e}(l=0) .
$$

Autrement dit, dans ces continuums élémentaires :

1) les résonances se traduisent par de fortes variations relatives des sections efficaces d'excitation et de la polarisation des raies $3{ }^{3} \mathrm{P}-2{ }^{3} \mathrm{~S}$ (interaction (1)) et $3{ }^{3} \mathrm{D}-2{ }^{3} \mathrm{P}$ (interaction (2)), (observation dans le canal photons).

2) les directions privilégiées pour observer ces résonances dans le canal électrons diffusés (canal e) doivent être voisines de $0^{\circ}$ (diffusion en avant) ou de $180^{\circ}$ (rétrodiffusion).

Les mesures dans le canal e ont été effectuées vers $0^{\circ}$ par Roy et al. [3] pour de nombreux continuums du groupe *. Sous grand angle, il ne semble pas qu'on ait étudié ces résonances au-delà de $50^{\circ}$ depuis les premiers travaux de Simpson et al. [6]. Outre l'intérêt fondamental que présentent ces résonances par les phénomènes fortement anisotropes qu'elles induisent, leur étude constitue un test pour la méthode exposée.

\section{Dispositif expérimental.}

Le dispositif expérimental est schématisé sur la figure 1. Le volume de collision est défini par le croisement à $90^{\circ}$ d'un faisceau supersonique d'hélium et d'un faisceau d'électrons l'un et l'autre à symétrie plane. Le détecteur de métastables $\mathrm{He}^{*} 2^{1,3} \mathrm{~S}$, centré sur la zone de collision figurée par le point $\mathrm{O}$, se déplace dans un plan contenant la direction des deux faisceaux $\mathrm{Ox}, \mathrm{Oz}$ (plan de collision).

Le faisceau supersonique est une version améliorée du modèle initial mis au point au laboratoire [7]. Il est du type basse pression à écorceur rectangulaire. La section du faisceau dans la zone de collision est $15 \times 1,5 \mathrm{~mm}$. Pour l'hélium, les caractéristiques principales sont :

- divergence $: 10^{-2}$ radian en $z, 10^{-1}$ radian en $y$,

- débit : $10^{20}$ atomes $\mathrm{s}^{-1} \mathrm{sr}^{-1}$,

— nombre de Mach : 16 . 


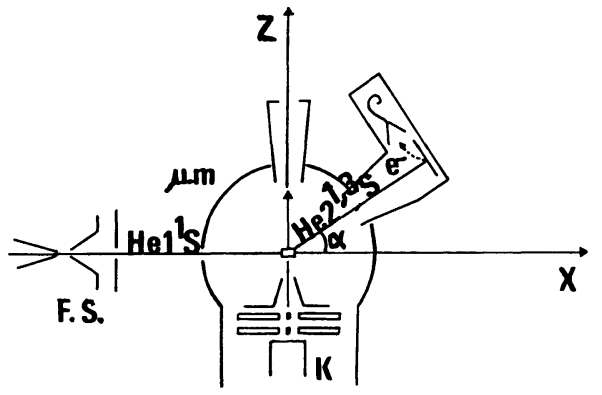

Fig. 1. - Schéma du dispositif expérimental. $\mathrm{K}$ est la cathode du canon à électrons. $\mu$-m est un écran en mu-métal.

[Diagram of the experimental set up. $\mathrm{K}$ is the cathode of the electron gun. $\mu-\mathrm{m}$ is a $\mu$-metal shield.]

Le canon à électrons également mis au point au laboratoire est du type de celui utilisé dans les expériences d'excitation [5]. C'est une rampe de $28(14 \times 2)$ canons élémentaires à symétrie cylindrique, dirigée le long de l'axe $y$; chaque élément donne un faisceau de $1 \mathrm{~mm}$ de diamètre et d'intensité typique $5 \mu \mathrm{A}$; la résolution à long terme (1 semaine) est meilleure que $0,3 \mathrm{eV}$. Le canon fonctionne soit en continu soit en impulsions de largeur minimum $10 \mathrm{~ns}$.

Le détecteur de métastables est constitué par l'association d'un convertisseur plan à couche d'or et d'un multiplicateur tubulaire d'électrons. Pour éviter les erreurs de parallaxe liées à l'utilisation d'une source allongée, un sélecteur compartimenté divise la zone observée en 7 volumes élémentaires de $2 \times 2 \times 1,5 \mathrm{~mm}$; l'élément correspondant sur le convertisseur, situé à $90 \mathrm{~mm}$, est un rectangle de $3 \times 4 \mathrm{~mm}$. L'ensemble sélecteur + détecteur est monté sur un bras mobile.

L'utilisation de faisceaux larges complique la réalisation du détecteur mais à résolution égale permet d'augmenter considérablement la quantité d'informations recueillie par unité de temps.

L'ensemble canon, volume de collision et détecteur est blindé par des écrans en mu-métal.

Une chaîne de coïncidence à deux voies avec convertisseur temps amplitude et analyseur multicanal permet d'obtenir le spectre des temps de vol des métastables produits par un faisceau pulsé d'électrons.

\section{Résultats.}

3. 1 TRANSFERT DE L'IMPULSION. - La détermination du vecteur impulsion transféré à l'atome par l'électron est simple et relève de la mécanique classique [8]. Les faisceaux supersonique et électronique sont représentés respectivement par les axes $\mathrm{O} x$ et $\mathrm{O} z$. Le vecteur $\mathrm{OA}$ représente l'impulsion d'un atome avant le choc. A l'instant du choc l'atome d'hélium à l'état fondamental absorbe l'impulsion $\mathbf{A B}$ d'un électron d'énergie $E$, puis l'électron incident ou échangé transfère à cet atome une impulsion $\mathbf{B C}$, en diffusant dans une direction quelconque de l'espace et en le laissant dans un état excité à un niveau d'énergie $E_{i}$. L'atome excité figuré par le vecteur impulsion $\mathrm{OC}$ est dévié d'un angle $\alpha$ par rapport à l'axe $\mathrm{Ox}$. Le détecteur est centré sur $\mathrm{O}$, centre d'un volume élémentaire de collision. Le faisceau atomique dévié est collimaté en angles $\alpha$ et $\phi(\mathbf{O C}$, $z \mathrm{Ox})$.

Ainsi, on détecte les métastables dans 7 plans élémentaires de collision représentés par le plan $z \mathrm{O} x: \phi=0,(\mathbf{O C}, \mathbf{O x})=\alpha($ Fig. 2). On note que pour une valeur de $\alpha$ on a deux valeurs différentes de $\theta$, sauf pour $\alpha_{\max }\left(\theta \simeq 148^{\circ}\right)$ et $\alpha_{\min }\left(\theta \simeq 0^{\circ}\right)$, correspondant à deux vagues de métastables, l'une accélérée par rapport à la vitesse initiale, l'autre ralentie. Ces vagues sont séparées par des mesures en temps de vol (Fig. 3). La résolution est insuffisante pour séparer 


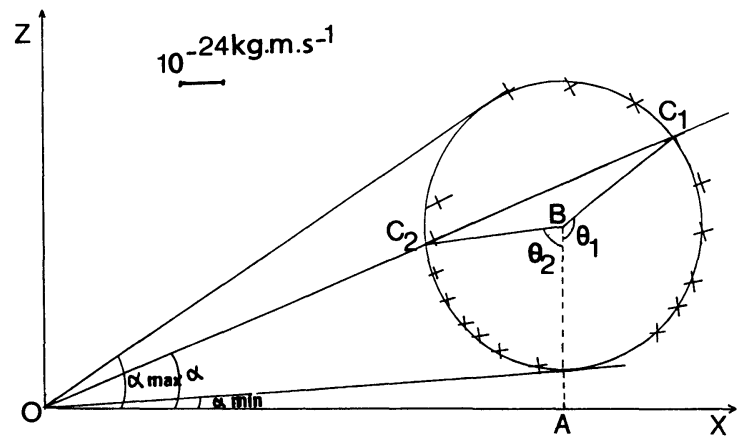

Fig. 2. - Transfert de l'impulsion dans le plan de collision. Nos résultats expérimentaux (croix) sont portés sur le cercle théorique.

[Momentum transfer in the collision plane. The present results (crosses) are plotted on the theoretical circle.]

les différents niveaux $E_{i}$. Sur le cercle théorique de rayon $\mathrm{BC}$ tracé avec $E_{i}=21 \mathrm{eV}\left(\mathrm{He}^{*} 2{ }^{3} \mathrm{P}\right)$ (Fig. 2), on a porté les mesures expérimentales de l'extrémité du vecteur $\mathbf{O C}$ déduites des mesures en temps de vol, en prenant pour chaque distribution la valeur la plus probable; les barres d'erreur représentent les incertitudes sur $\alpha$ et sur les temps de vol. Pour $\alpha>\alpha_{\max }$ et $\alpha<\alpha_{\min }$ on détecte seulement le rayonnement U.V. dû essentiellement à la transition $2{ }^{1} \mathrm{P}-2{ }^{1} \mathrm{~S}$. Ce rayonnement représente au maximum $10 \%$ du signal dû aux métastables.

3.2 OBSERVATION DES RÉSONANCES. - En toute rigueur, seules les mesures en temps de vol permettent de séparer $\theta_{1}$ et $\theta_{2}$. Cependant, les résultats de la figure 3 montrent que vers $\alpha_{\max }$ la deuxième vague de métastables est très atténuée du fait de la collimation en $\phi$. Comme dans

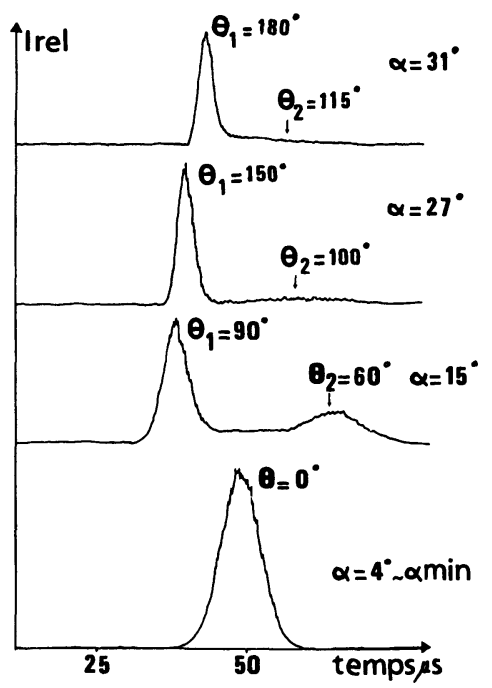

Fig. 3. - Spectre en temps de vol des métastables.

[Metastables time of flight spectrum.] 
une première étape nous n'avons cherché que des résultats indicatifs de la diffusion résonnante sous grands angles, nous n'avons pas utilisé le canon en mode pulsé pour observer les résonances afin de ne pas allonger les temps de mesure. Fn opérant ainsi on voit que la résolution en angle $\theta$ est limitée par la distribution initiale des vitesses du faisceau supersonique. La figure 4 montre le résultat des mesures; nous avons centré notre étude sur la résonance $\mathrm{He}^{-* * * 2} \mathrm{P}$. Sous différents angles cette résonance est bien représentée par des profils de Beutler-Fano décrits par la fonction non normalisée : $f(\varepsilon)=\frac{(q+\varepsilon)^{2}}{1+\varepsilon^{2}}$, où $\varepsilon$ est l'énergie réduite et $q$ le facteur de forme ; selon que $|q|$ augmente ou diminue à partir de la valeur $|q|=1$ la structure tend vers un pic parfait $|q \rightarrow \infty|$ ou vers un creux parfait $q=0$.

Pour $\theta \simeq 0^{\circ}$ la structure est en creux correspondant aux observations de Bolduc et Marmet [4]. La variation relative de la section efficace : $-2,5 \%$ à la résonance correspond aux $-8 \%$ trouvés par ces auteurs qui utilisent un faisceau d'électrons dont la résolution est meilleure que $0,1 \mathrm{eV}$.

Pour $\theta \simeq 180^{\circ}$ la structure est en pic d'intensité relative $+7 \%$.

Pour $\theta \simeq 90^{\circ}$ la structure est plus symétrique $(q \simeq-0,75)$ : à la résonance la variation relative de la section efficace est inférieure à $1 \%$.

On remarquera que la détection des métastables revient à détecter tous les niveaux triplets et le niveau $2{ }^{1} \mathrm{~S}$ avec un égal rendement. Pour le niveau $2{ }^{1} \mathrm{P}$, si les mesures de Roy et al. vers $0^{\circ}$ [3] indiquent la variation absolue la plus grande à la résonance, la variation relative est faible. $\mathrm{Si}$ on note également que : 1) cette résonance est en creux ; 2) la section efficace différentielle du niveau $2{ }^{1} \mathrm{P}$ diminue considérablement vers les grands angles [9], on peut dire que la résonance $\mathrm{He}^{-* * * 2} \mathrm{P}$ se manifeste par une diminution de la section efficace de diffusion inélastique des électrons vers l'avant $\left(\theta_{\min }\right)$ et une augmentation de la section efficace de rétrodiffusion $\left(\theta_{\max }\right)$.

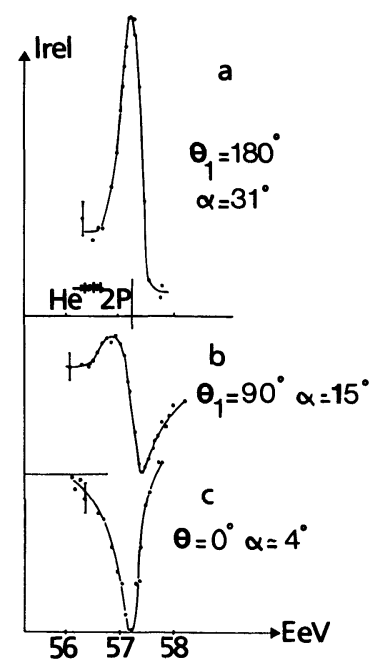

Fig. 4. - Fonction d'excitation des métastables à la résonance. Le fond non résonnant a été soustrait par approximation linéaire. Intensité relative des extréma par rapport au fond non résonnant : a) maximum $=+7 \%$; b) maximum $=+2 \%$, minimum $=-3,5 \%$; c) minimum $=-2,5 \%$. Les barres d'erreur indiquent l'erreur statistique.

[Excitation function of metastables at resonance. The non-resonant background has been subtracted in a linear approximation. Relative intensity of extrema by reference to the non-resonant background : a) maximum $=+7 \%$; b) maximum $=+2 \%$, minimum $=-3.5 \%$; c) minimum $=-2.5 \%$. The statistical error is indicated by an error bar.] 
L'effet relatif de la résonance pour la diffusion à $180^{\circ}$ peut être estimé, compte tenu des résultats de Chutjian et Srivastava [9], à environ $+10 \%$ tous niveaux confondus en se basant sur une largeur de la résonance de $90 \mathrm{meV}$ [4].

Les interférences entre les différentes probabilités de transition sont complexes. Néanmoins, l'ensemble de nos observations et celles des auteurs cités peuvent se résumer ainsi, pour les phénomènes dominants, à la résonance :

- la transition de l'état initial e $(\theta=0)+\mathrm{He}$ vers l'état lié $\mathrm{He}^{-* * *}{ }^{2} \mathrm{P}$ se fait au détriment de la transition de l'état initial vers le continuum e $\left(\theta_{\min }\right)+\mathrm{He}^{*}$ (singulet et principalement $\left.2{ }^{1} \mathrm{P}[3]\right)$, d'où des structures en creux ;

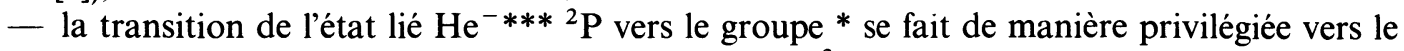
continuum e $\left(\theta_{\max }\right)+\mathrm{He}^{*}$ (vraisemblablement triplet $2{ }^{3} \mathrm{P}$ ) (nos observations) et vers le continuum e $\left(\theta_{\min }\right)+\mathrm{He}^{*}\left(2^{3} \mathrm{P}\right)$ [3], d'où des structures en pic.

\section{Conclusion.}

Nous avons montré la validité d'une méthode qui permet, lorsqu'elle s'applique, de s'affranchir outre de l'encombrement géométrique, des électrons parasites et des champs magnétique et électrique parasites. Sans traitement numérique approprié, la fonction d'appareil introduite n'autorise pas des mesures précises. Cependant, nous avons pu faire des observations intéressantes.

L'interaction du niveau $\mathrm{He}^{-* * *}{ }^{2} \mathrm{P}$ sur le groupe de continuums $\mathrm{He}^{*}+\mathrm{e}$ s'observe comme une dissymétrie de la diffusion des électrons par rapport au centre de collision. A la résonance, la décomposition de l'ion négatif (transition non radiative) se traduit apparemment par une éjection privilégiée d'électrons dans le sens inverse du faisceau incident, alors que dans le processus d'excitation directe la diffusion inélastique des électrons vers l'avant domine largement. Pour mesurer une éventuelle dissymétrie de l'éjection d'électrons par rapport à un centre, il faut travailler sur un seul continuum du groupe *. Nous nous proposons, dans l'étape suivante, d'étudier les interactions (1) et (2) par des expériences de coïncidences photon/métastable; à savoir : $h v\left(\mathrm{He}^{*} 3{ }^{3} \mathrm{P}-2{ }^{3} \mathrm{~S}\right) / \mathrm{He}^{*} 2{ }^{3} \mathrm{~S}$ pour l'interaction (1) et $h v\left(\mathrm{He} * 3{ }^{3} \mathrm{D}-2{ }^{3} \mathrm{P}\right) / \mathrm{He} * 2{ }^{3} \mathrm{~S}$ pour l'interaction (2). Théoriquement le meilleur taux de coïncidences serait obtenu avec $h v\left(\mathrm{He}^{*} 3^{3} \mathrm{D}\right.$ $\left.2{ }^{3} \mathrm{P}\right) / \mathrm{He}^{*} 2^{3} \mathrm{~S}$; malheureusement les photomultiplicateurs actuels ont un rendement trop faible pour la longueur d'onde correspondant à la transition $2{ }^{3} \mathrm{P}-2{ }^{3} \mathrm{~S}(1080 \mathrm{~nm})$.

\section{Bibliographie}

[1] Rubin, K., Perel, J. and Bederson, B., Phys. Rev. 117 (1960) 151.

[2] Van Ittersum, T., Heideman, H. G. M., Nienhuis, G. and Prins, J., J. Phys. B 9 (1976) 1713.

[3] Roy, D., Delâge, A. and Carette, J. D., J. Phys. B 11 (1978) 4059.

[4] Bolduc, E. and Marmet, P., J. Phys. B 8 (1975) L241.

[5] Defrance, A., J. Phys. B 13 (1980) 1229.

[6] Simpson, J. A., Menendez, M. G., Mielczareck, S. R., Phys. Rev. 150 (1966) 76.

[7] Treguier, J. P., Hagene, M., Pasquerault, D., Revue Phys. Appl. 5 (1970) 343.

[8] Lamb, W. E., Retherford, R. C., Phys. Rev. 79 (1950) 549.

[9] Chutuin, A. and Srivastava, S. K., J. Phys. B 8 (1975) 2360. 